\title{
CORRECTION
}

\section{Correction to: Early EEG for Prognostication Under Venoarterial Extracorporeal Membrane Oxygenation}

Eric Magalhaes ${ }^{1 \dagger}$, Jean Reuter ${ }^{1,2+}$, Ruben Wanono ${ }^{3 \dagger}$, Lila Bouadma $^{1,4}$, Pierre Jaquet ${ }^{1}$, Sébastien Tanaka ${ }^{5,6}$, Fabrice Sinnah', Stéphane Ruckly ${ }^{4}$, Claire Dupuis ${ }^{1,4}$, Etienne de Montmollin ${ }^{1,4}$, Marylou Para ${ }^{7}$, Wael Braham Angelo Pisani ${ }^{7}$, Marie-Pia d'Ortho ${ }^{3}$, Anny Rouvel-Tallec ${ }^{3}$, Jean-François Timsit ${ }^{1,4}$, Romain Sonneville ${ }^{1,2^{*}}$ (D) and DINAMO study group

(C) 2020 Springer Science+Business Media, LLC, part of Springer Nature and Neurocritical Care Society

\section{Correction to: \\ Neurocrit Care \\ https://doi.org/10.1007/s12028-020-01066-3}

The original article was updated by replacing an incorrect institutional author to DINAMO study group towards the end of the author list. Table 1 was updated with modifications on the lines "History of acute brain injury" and "At ICU admission".

Acknowledgements section is corrected below to have the DINAMO study group information added towards the end.

\footnotetext{
Author details

${ }^{1}$ Department of Intensive Care Medicine, AP-HP, Bichat-Claude Bernard Hospital, 46 Rue Henri Huchard, 75018 Paris Cedex, France. ${ }^{2}$ INSERM UMR1148, Team 6, Université de Paris, 75018 Paris, France. ${ }^{3}$ Department of Physiology, AP-HP, Bichat-Claude Bernard Hospital, 75018 Paris, France. ${ }^{4}$ UMR 1137, IAME, Université de Paris, Paris, France. ${ }^{5}$ Department of Anesthesiology and Intensive Care, AP-HP, Bichat-Claude Bernard Hospital, 75018 Paris, France. ${ }^{6}$ INSERM 1188, DéTROI, Reunion Island University, Saint-Denis de la Réunion, France. ${ }^{7}$ Department of Cardiac Surgery, AP-HP, Bichat-Claude Bernard Hospital, 75018 Paris, France.
}

\begin{abstract}
Acknowledgements
The authors wish to thank the following collaborators: Nadine Ajzenberg: Department of Immunology and Hematology, AP-HP, Bichat-Claude Bernard Hospital, Paris, France; Marie-Charlotte Bourrienne, Department of Immunol ogy and Hematology, AP-HP, Bichat-Claude Bernard Hospital, Paris, France; Claire Dupuis: Department of Intensive Care Medicine, AP-HP, Bichat-Claude Bernard Hospital, Paris, France; Dorothée Faille: Department of Immunology and Hematology, AP-HP, Bichat-Claude Bernard Hospital, Paris, France; Mikael Mazighi: Department of Neurology, AP-HP, Lariboisière hospital, Paris, France; Patrick Nataf: Department of cardiac surgery, AP-HP, Bichat-Claude Bernard Hospital, France; and Katell Peoc'h: Department of Biochemistry, Beaujon University Hospital, AP-HP, Bichat-Claude Bernard Hospital, Clichy, France; DINAMO is the original acronym of the study group that stands for "Developing Neuroprognostication in Adults under ECMO".
\end{abstract}

\section{Publisher's Note}

Springer Nature remains neutral with regard to jurisdictional claims in published maps and institutional affiliations.

Published online: 9 October 2020

*Correspondence: romain.sonneville@aphp.fr

†Eric Magalhaes, Jean Reuter, and Ruben Wanono have contributed equally to this work

${ }^{1}$ Department of Intensive Care Medicine, AP-HP, Bichat-Claude Bernard

Hospital, 46 Rue Henri Huchard, 75018 Paris Cedex, France 